\title{
Physical-chemical and chemical analysis of groundwater of Monsarás and Condeixa communities, Salvaterra-PA
}

\author{
Emerson Renato Maciel da Silva ${ }^{1}$; Andrey Nildo de Jesus da Luz Souza Junior ${ }^{2}$; Samya Uchôa Bordallo ${ }^{3}$; \\ Regina Celi Sarkis Muller ${ }^{4}$; Ewerton Carvalho de Souza ${ }^{5}$; Ivan Carlos da Costa Barbosa ${ }^{6}$
}

1,2,3,5,6 Universidade Federal Rural da Amazônia (UFRA). Avenida Presidente Tancredo Neves, No 2501 Bairro: Terra Firme. CEP: 66.077-830. Cidade: Belém-Pará-Brasil. Telefone: +55 (91) 9-825 38691.

${ }^{4}$ Programa de Pós-Graduação em Ciências e Meio Ambiente do Instituto de Ciências Exatas e Naturais da Universidade federal do Pará (ICEN-UFPA). Rua Augusto Corrêa, 01 - Guamá. CEP: 66075-110. Caixa postal 479. Cidade: Belém-Pará-Brasil. Telefone: +55 (91) 991342265.

Email:emersonrvs255@gmail.com,andreynildo@gmail.com,samyauchoa2000@gmail.com,reginasarkis@hotmail.com, ewcarvalho@yahoo.com.br, belemivan@hotmail.com

Received: January $10^{\text {th }}, 2017$

Accepted: February $21^{\text {th }}, 2017$

Published: March 30th, 2017

Copyright $\odot 2016$ by authors and Institute of Technology Galileo of Amazon (ITEGAM). This work is licensed under the Creative Commons Attribution International License (CC BY 4.0).

http://creativecommons.org/licenses/ by/4.0/ (c) (i) (9) Open Actes:

\begin{abstract}
One of the water sources for consumption that has been used a lot is Amazonas wells, as an cheap water source. Based on this assumption, this study aimed to evaluate the physical-chemical and

results with the maximum values allowed by Ordinance 2,914/11. The instrumental and volumetric methods were used to determine the physic-chemical and chemical parameters. Subsequently, the statistical treatment was performed through multivariate analysis. The results revealed that the parameters meet the limits established by the Ordinance, except the turbidity in Condeixa in June to August. The parameters that was not recommended by the legislation contributed significantly to establish the quality of contamination from anthropogenic actions. The use of multivariate analysis contributed to obtain important information in the identification of similarities and differences between the parameters studied.
\end{abstract}

Key-Words: Amazonas wells, Groundwater, Multivariate analysis.

\section{Análise físico-química e química de águas subterrâneas das comunidades de Monsarás e Condeixa, Salvaterra-PA}

\begin{abstract}
RESUMO
Uma das fontes de água para consumo que tem sido muito utilizada são os poços amazonas, por ser uma fonte hídrica economicamente barata. Partindo desse pressuposto, o presente estudo objetivou avaliar a qualidade físico-química e química das águas de poços nas comunidades de Monsarás e Condeixa. Assim como comparar os resultados com os valores máximos permitidos pela portaria 2.914/11. Para a determinação dos parâmetros físico-químicos e químicos foram empregados os métodos instrumental e volumétrico. Posteriormente foi realizado o tratamento estatístico através da análise multivariada. Os resultados revelaram que os parâmetros atendem os limites estabelecidos pela portaria, exceto a turbidez em Condeixa nos meses de junho a agosto. Os parâmetros não preconizados pela legislação contribuíram de forma significativa para estabelecer a qualidade quanto à contaminação proveniente de ações antrópicas. A utilização da análise multivariada contribuiu para a obtenção de informações importantes na identificação de semelhanças e diferenças para os parâmetros estudados.
\end{abstract}

Palavras-Chave: poços amazonas, águas subterrâneas, análise multivariada.

\section{INTRODUÇÃO}

A água é um recurso natural considerado inevitável para o desenvolvimento das atividades humanas, assim como, um elemento bioquímico dos seres vivos, e indispensável para os meios vegetais e animais, além de operar como fator de produção para vários bens de consumo primário e secundário [1,2]. Mesmo 
estando em quantidade elevada no planeta, somente $0,8 \%$ encontrase disponível para ingestão humano. Sua disponibilidade é comprometida por meio de contaminações, resultando numa exploração maior dos recursos hídricos subterrâneos [3].

Dentre as inúmeras fontes para obtenção de água, podemos destacar a água subterrânea derivadas de poços amazonas. Essas águas têm sido cada vez mais utilizadas para o consumo humano, pois além de ser economicamente barata é uma fonte hídrica de fornecimento imprescindível para as pessoas que não tem acessibilidade a rede pública de abastecimento de água $[4,5]$.

Do ponto de vista técnico, as águas subterrâneas e superficiais são as mais propensas à contaminação por produtos químicos. Em comunidades agrícolas, o uso de agrotóxicos sem acompanhamento técnico pode comprometer a qualidade da água, causando extrema preocupação, principalmente quando a mesma é usada para o consumo humano [6]. Em consequência disso, o uso desses produtos químicos podem aumentar a concentração do nível de nitrato e fosfato fazendo com que essa água se torne imprópria [7].

Esse tipo de fornecimento de água é muito preocupante, devido as formas como são feitas e por não terem nenhum planejamento, quanto a distância de locais onde são descartados lixo, excremento humano e plantio de culturas agrícolas [5]. Indiscutivelmente essa má conservação, higienização e manejo inadequado desses poços que armazenam essa água, podem desencadear alterações dos padrões físico-químicos causados pela presença de fatores contaminantes e comprometendo a qualidade da água. Tais fatores são fundamentais para problemas de saúde pública [8].

Para que a água subterrânea seja considerada potável, é imprescindível a verificação por meio das análises físico-químicas, químicas e microbiológicas, a fim de constatar se ela está inclusa nos padrões de potabilidade para consumo humano instituídos nos regulamentos vigentes em cada País $[9,10]$.

O Ministério da Saúde por meio da portaria $n^{\circ} 2.914$ de 2011, estabelece que a água potável destinada para a ingestão humana precisa ter os parâmetros físico-químicos, microbiológicos e radioativos aceitáveis ao padrão de potabilidade e que não proporcione riscos à integridade humana. Quanto aos parâmetros organolépticos, estes não podem apresentar sabor e nem odor indesejáveis. Os parâmetros físicos precisam ter valores instituídos dentro dos limites estabelecidos de potabilidade. E os parâmetros químicos não podem conter substâncias tóxicas ou nocivas acima dos limites tolerados pelo homem. Os parâmetros microbiológicos não podem conter micro-organismos transmissores de doenças [11].

Portanto, este trabalho teve como objetivo avaliar a qualidade físico-química e química das águas subterrâneas oriundas de poços artesianos ou amazonas, nas comunidades de Condeixa e Monsarás em Salvaterra, situada no arquipélago do Marajó-PA. Assim como comparar os resultados com os valores máximos permitidos pela portaria 2.914/11 do Ministério da Saúde.

\section{MATERIAL E MÉTODOS}

A pesquisa foi realizada nas comunidades de Condeixa e Monsarás, distritos do município de Salvaterra, localizado na Mesorregião do Marajó, pertencente à Microrregião do Arari no estado do Pará. O município está situado nas coordenadas geográficas $00^{\circ} 45^{\prime} 21^{\prime \prime}$ de latitude sul e $48^{\circ} 45^{\prime} 54^{\prime \prime}$ de longitude oeste de Greenwich. O município de Salvaterra possui um contingente populacional de acordo com o censo 2010 do Instituto Brasileiro de Geografia e Estatística de 20.183 habitantes, onde cerca de 3,93\% (793 habitantes) pertencem à comunidade de Monsarás, e aproximadamente $12,74 \%$ (2.572 habitantes) à comunidade de Condeixa [12].

Inicialmente, foi realizada uma visita prévia nas comunidades de Condeixa e Monsarás, onde foram selecionadas dez residências, sendo cinco em Monsarás, e cinco em Condeixa, tendo como critério de seleção as casas que utilizem água de poços amazonas (Figura 1).

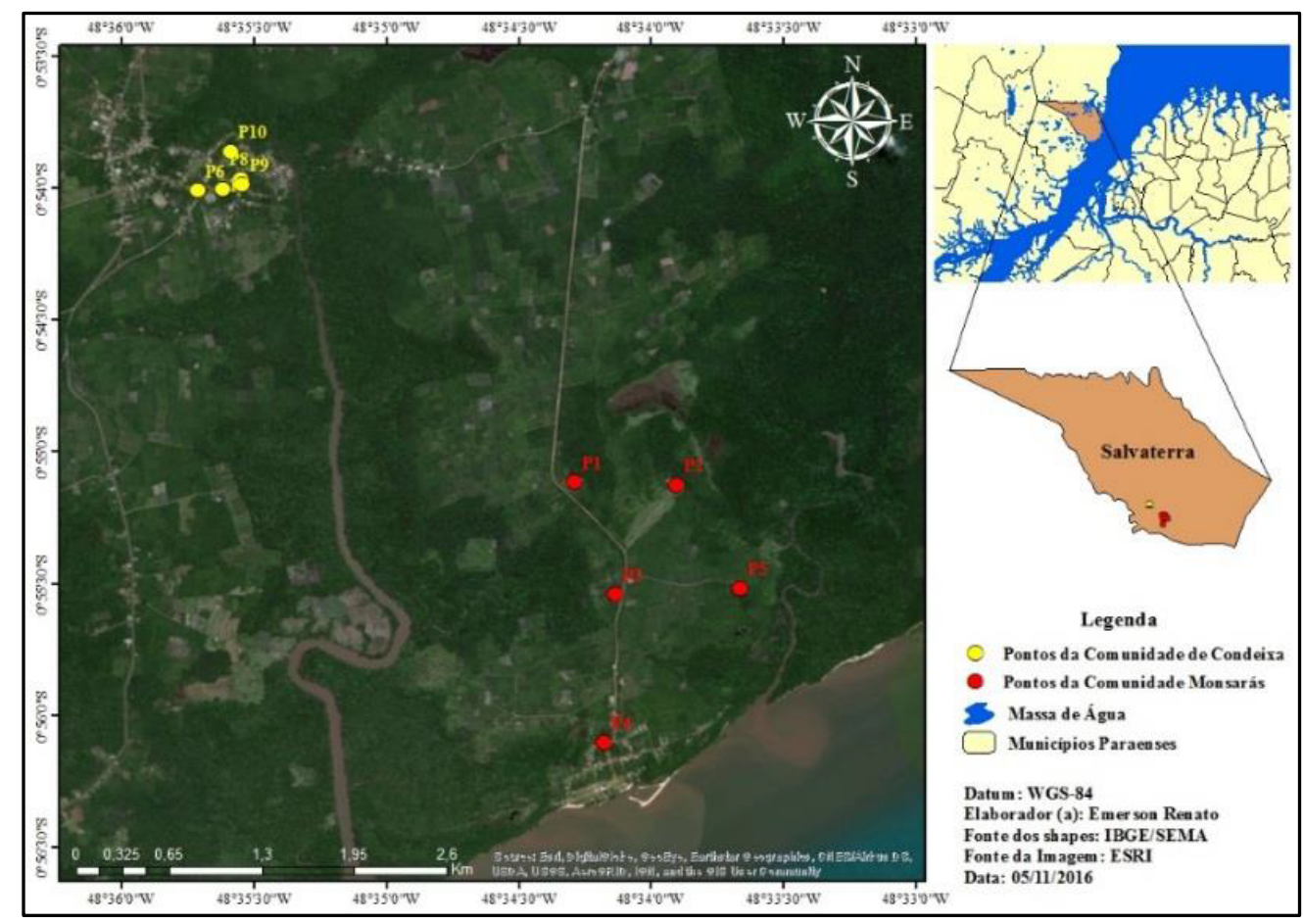

Figura 1: Mapa de localização da área de estudo. 
Após esse procedimento foram executadas as coletas das amostras das águas subterrâneas (poços). Um balde "virgem", previamente lavado no laboratório com água e sabão foi utilizado como ferramenta de coleta da água. No local de coleta foi realizada a limpeza do balde com água destilada, e a ambientalização com a água do poço a ser analisada. Este procedimento foi necessário a fim de evitar contaminação de fatores externos.

Cada amostra foi armazenada em dois frascos plásticos de polietileno com capacidade de $1000 \mathrm{~mL}$ cada. Os frascos foram antecipadamente limpos e esterilizados, devidamente identificados e acondicionados em uma caixa térmica com gelo, visando assim, tornar mínima a contaminação por fatores abióticos ou externos até as análises no laboratório de Físico-Química no Centro de Tecnologia Agropecuária (CTA) da Universidade Federal Rural da Amazônia (UFRA). Todas as determinações foram realizadas em triplicata para se obter uma média e um desvio-padrão acessíveis, para que fosse possível comparar os resultados e aplicar a Análise de Componentes Principais (PCA) e a Análise Hierárquica de Cluster (HCA).

\section{II.1 ANÁLISES IN SITU}

No local de coleta, foram realizadas as análises dos parâmetros físico-químicos: potencial hidrogeniônico (pH), condutividade elétrica (CE), oxigênio dissolvido (OD) e turbidez (uT).

$\mathrm{O} \mathrm{pH}$ (adimensional) foi aferido em todas as amostras, utilizando-se um peagâmetro de campo, Digital Instruments ${ }^{\circledR}$, modelo PH-1900, devidamente calibrado com soluções tampão de pH 7,0 e 4,0.

A determinação da condutividade elétrica, expressa em $\mu \mathrm{S}$ $\mathrm{cm}^{-1}$, foi realizada por meio de um condutivímetro portátil (Instrutherm ${ }^{\circledR}, \mathrm{CD}-850$ ), previamente calibrado com solução padrão de $1413 \mu \mathrm{S} \mathrm{cm} \mathrm{cm}^{-1}$.

As análises de oxigênio dissolvido, expresso em $\mathrm{mg} \mathrm{L}^{-1}$, foram realizadas por meio de um oxímetro portátil Instrutherm ${ }^{\circledR}$, modelo MO-910, previamente calibrado.

As análises de turbidez, expressa em unidades de turbidez (uT), foram realizadas por meio do aparelho turbidímetro portátil Instrutherm ${ }^{\circledR}$, modelo TD-300, devidamente calibrado com soluções padrão de 0 NTU e 100 NTU.

\section{II.2 ANÁLISES LABORATORIAIS}

No laboratório foram feitas as análises dos parâmetros químicos: cloreto $\left(\mathrm{Cl}^{-}\right)$, acidez (ACD), alcalinidade (ALC), dureza total (DT) e resíduos totais (RT). Todos os métodos empregados seguem como referência as metodologias descritas na $21^{\mathrm{a}}$ edição do Standart Methods for the Examination of Water and Wastewater [13] (APHA, 2005).

A determinação do teor de cloreto, expresso em $\mathrm{mg} \mathrm{de} \mathrm{Cl}^{-}$ $\mathrm{L}^{-1}$, foi obtida por meio do método $4500 \mathrm{~B}$, volumetria de precipitação, também comumente conhecida como método de Mohr.

Para determinação da acidez, expressa em $\mathrm{mg} \mathrm{CaCO}_{3} \mathrm{~L}^{-1}$, foi empregado método 2310B, titulação com indicador, utilizando o hidróxido de sódio $(\mathrm{NaOH})$ como titulante e a fenolftaleína como indicador.

Para determinação da alcalinidade, expressa em mg $\mathrm{CaCO}_{3} \mathrm{~L}^{-1}$, foi empregado método $2320 \mathrm{~B}$, utilizando o ácido sulfúrico $\left(\mathrm{H}_{2} \mathrm{SO}_{4}\right)$ como titulante e o verde de bromocresol como indicador.

Para determinação da dureza total, expressa em $\mathrm{mg} \mathrm{L}^{-1} \mathrm{de}$ $\mathrm{CaCO}_{3}$, foi utilizado o método $2340 \mathrm{C}$, volumetria de complexação, que tem a finalidade de determinar concentrações de íons metálicos, utilizando o EDTA (etilenodiaminotetracético) como titulante. Foi utilizado o indicador EBT (negro de Eriocromo T) como indicador.

Para determinação de resíduos totais foi utilizado o método gravimétrico. O qual se determina por meio da diferença entre massas, ou seja, a diferença entre a massa seca e a massa úmida, em relação ao volume de alíquota utilizado.

\section{RESULTADOS E DISCUSSÕES}

Para as análises estatísticas, foram calculadas as médias gerais dos cincos pontos estudados para cada mês. Assim obtevese a média geral e desvio padrão geral dos resultados para as coletas realizadas no mês de junho para Monsarás e Condeixa, denominados de MJU e CJU, no mês de julho MJL e CJL e no mês de agosto MAG e CAG.

Os resultados referentes aos parâmetros físico-químicos estão apresentados na tabela 1 .

Tabela 1: Resultados dos parâmetros físico-químicos, assim como as respectivas médias e desvios padrão.

\begin{tabular}{c|c|c|c|c}
\hline Comunidades & $\mathbf{p H}$ & $\begin{array}{c}\text { OD } \\
\left(\mathbf{m g ~ L}^{-\mathbf{1}}\right)\end{array}$ & $\begin{array}{c}\mathbf{C E} \\
\left(\boldsymbol{\mu S} \mathbf{~ c m}^{-\mathbf{1}}\right)\end{array}$ & $\begin{array}{c}\text { TRB } \\
(\mathbf{u T})\end{array}$ \\
\hline MJU & $8,63 \pm 0,69$ & $6,72 \pm 1,75$ & $84,70 \pm 73,58$ & $2,31 \pm 1,49$ \\
\hline MJL & $8,16 \pm 0,91$ & $6,66 \pm 1,90$ & $68,20 \pm 50,57$ & $3,51 \pm 2,96$ \\
\hline MAG & $7,76 \pm 0,79$ & $5,69 \pm 2,61$ & $62,20 \pm 37,92$ & $3,99 \pm 3,86$ \\
\hline CJU & $8,55 \pm 0,37$ & $4,54 \pm 1,77$ & $204,00 \pm 145,01$ & $7,91 \pm 14,80$ \\
\hline CJL & $7,91 \pm 1,46$ & $5,93 \pm 1,33$ & $121,13 \pm 89,06$ & $106,11 \pm 231,00$ \\
\hline CAG & $7,99 \pm 1,06$ & $6,34 \pm 2,86$ & $145,87 \pm 83,04$ & $70,52 \pm 153,26$ \\
\hline VMP* & $\mathbf{6 , 0} \mathbf{9} \mathbf{9 , 5}$ & - & - & $\mathbf{5} \mathbf{~ u T}$ \\
\hline
\end{tabular}

Nota: *VMP: valor máximo permitido pela Portaria $\mathrm{n}^{\circ} 2.914 / 2011$.

Em relação ao pH, observa-se que no mês de junho, tanto na comunidade de Monsarás quanto Condeixa, foram obtidos os maiores resultados. Sendo que, em todos os meses, os resultados encontraram-se dentro dos padrões de potabilidade prescritos pela
Portaria $\mathrm{n}^{\circ}$ 2.914/2011 do Ministério da Saúde, que atribui como VMP a faixa de 6,0 a 9,5 [11].

Com relação à concentração de oxigênio dissolvido, os valores não são preconizados pela portaria 2.914/2011. No entanto 
este parâmetro é importante não apenas nos processos de respiração da fauna aquática, como também no processo de desnitrificação e biodegradação de contaminantes [14].

A condutividade elétrica determina a capacidade da água de conduzir corrente elétrica, estando totalmente ligada com o teor de sais dissolvidos sob forma de íons. A condutividade elétrica apresentou maiores valores na comunidade de Condeixa, variando em relação aos meses de Julho e Junho respectivamente $121,13 \mu \mathrm{S}$ $\mathrm{cm}^{-1}$ à $204,00 \mu \mathrm{S} \mathrm{cm}^{-1}$. A legislação em comparação não apresenta valores máximos permitidos para avaliar a condutividade elétrica.

Na comunidade de Monsarás, os resultados para turbidez, em todos os meses, apresentaram-se dentro do VMP pela legislação, que é de 5,0 uT, variando de 2,31 uT no mês de junho a 3,99 uT no mês de agosto. Porém, quando comparado aos valores obtidos na comunidade de Condeixa, observa-se um aumento acentuado deste parâmetro de 7,91 uT a 106,11 uT. A turbidez está relacionada à quantidade de particulas em suspensão. Conjecturase que essa extrema variação esteja associada ao fato de alguns poços estarem em um local bem arborizado e não conterem proteção, e outros estarem sofrendo processo de erosão, além de não conterem proteção adequada.

A tabela 2 apresenta os resultados obtidos para os parâmetros químicos, assim como as médias gerais e desvios padrão.

Tabela 2: Resultados dos parâmetros químicos, assim como as respectivas médias e desvios padrão.

\begin{tabular}{|c|c|c|c|c|c|}
\hline Comunidades & 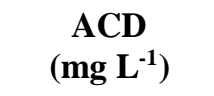 & 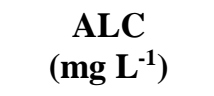 & $\begin{array}{c}\mathrm{Cl}^{-} \\
\left(\mathrm{mg} \mathrm{L}^{-1}\right)\end{array}$ & $\begin{array}{c}\text { DT } \\
\left(\mathbf{m g ~ L ^ { - 1 }}\right)\end{array}$ & 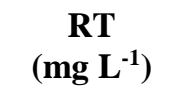 \\
\hline MJU & $49,20 \pm 17,58$ & $7,00 \pm 4,74$ & $40,97 \pm 16,15$ & $8,27 \pm 3,29$ & $0,03 \pm 0,03$ \\
\hline MJL & $35,45 \pm 15,73$ & $8,80 \pm 2,64$ & $23,33 \pm 11,99$ & $13,65 \pm 2,98$ & $0,10 \pm 0,06$ \\
\hline MAG & $20,39 \pm 10,54$ & $22,27 \pm 24,54$ & $37,97 \pm 10,08$ & $11,20 \pm 2,19$ & $0,05 \pm 0,05$ \\
\hline CJU & $50,68 \pm 20,16$ & $21,87 \pm 27,63$ & $50,87 \pm 23,22$ & $25,73 \pm 29,21$ & $0,05 \pm 0,05$ \\
\hline CJL & $54,70 \pm 24,95$ & $17,74 \pm 21,86$ & $25,19 \pm 8,62$ & $25,53 \pm 20,94$ & $0,18 \pm 0,14$ \\
\hline CAG & $29,39 \pm 16,10$ & $22,60 \pm 21,81$ & $39,34 \pm 10,30$ & $27,44 \pm 23,96$ & $0,18 \pm 0,16$ \\
\hline VMP* & - & - & $250 \mathrm{mg} \mathrm{L}^{-1}$ & $500 \mathrm{mg} \mathrm{L}^{-1}$ & - \\
\hline
\end{tabular}

Com relação à acidez, pode-se observar que os valores mais elevados foram obtidos nos meses de junho e julho na comunidade de Condeixa. Sua determinação é importante, uma vez que indica lançamentos de resíduos industriais no corpo hídrico, quando apresenta elevados valores. Para este parâmetro não existem valores estabelecidos pela portaria 2.914/2011.

No mês de agosto houve um aumento no teor de alcalinidade nas amostras de água das duas comunidades. A legislação vigente não preconiza valores máximos permitidos para esse parâmetro. A sua avaliação é relevante no processo de tratamento da água, pois é em função da sua concentração que se determina a quantidade de substâncias químicas a serem adicionadas na água [15].

Nos resultados das concentrações de cloreto, pode-se perceber que nos pontos estudados o teor oscilou em todos os meses, apresentando como maior concentração no mês de junho na comunidade de Condeixa (CJU). Quando ultrapassa o limite permitido pela Portaria $\mathrm{n}^{\circ} 2.914 / 2011 \mathrm{de} 250 \mathrm{mg} \mathrm{L}^{-1}$ proporciona sabor salgado, o que leva a rejeição pelo consumidor. Todavia, essa oscilação não ultrapassou o valor máximo permitido de $250 \mathrm{mg} \mathrm{L}$ 1 determinados pela portaria $\mathrm{n}^{\mathrm{o}} 2.914 / 2011$. Todos os pontos apresentaram valores abaixo do valor máximo permitido [11].

De acordo com a Portaria $n^{\circ} 2.914 / 2011$, o valor máximo permitido para dureza total é de $500 \mathrm{mg} \mathrm{L}^{-1}$. A utilização da água com uma dureza acima de $200 \mathrm{mg} \mathrm{L}^{-1}$ proporciona problemas relacionados à incrustações em tubulações [16]. Foi observado que os maiores valores obtidos encontram-se nas amostras da comunidade de Condeixa com uma média de $26,23 \mathrm{mg} \mathrm{L}^{-1}$ no período estudado, e uma média de $11,04 \mathrm{mg} \mathrm{L}^{-1}$ na comunidade de Monsarás.

Para resíduos totais, os resultados obtidos variaram nos meses da referida pesquisa. Os meses de julho e agosto da comunidade de Condeixa apresentaram os maiores valores em relação aos demais pontos. Isso pode estar associado à erosão, como o fenômeno que influencie o aumento da turbidez. A legislação vigente não preconiza os valores máximos permitidos para os resíduos totais.

\section{III.1 ANÁLISE MULTIVARIADA}

Com o objetivo de se obter uma melhor interpretação dos dados, resultantes das análises físico-químicas e químicas, aplicouse a técnica multivariada de análise de componentes principais (PCA), a qual inicialmente levou a projeção dos dados em um espaço com 9 dimensões, ou seja, nove componentes principais, mas apenas as três componentes iniciais (PC1: 39,7\%, PC2: 33,4\%, PC3: $19,8 \%$ ), foram as mais significativas na explicação das variações dos dados, totalizando uma explicação de aproximadamente $92,9 \%$ dessa variação. Desta forma obteve-se o gráfico de score por meio da projeção da PC1 x PC2, conforme a Figura 2. 


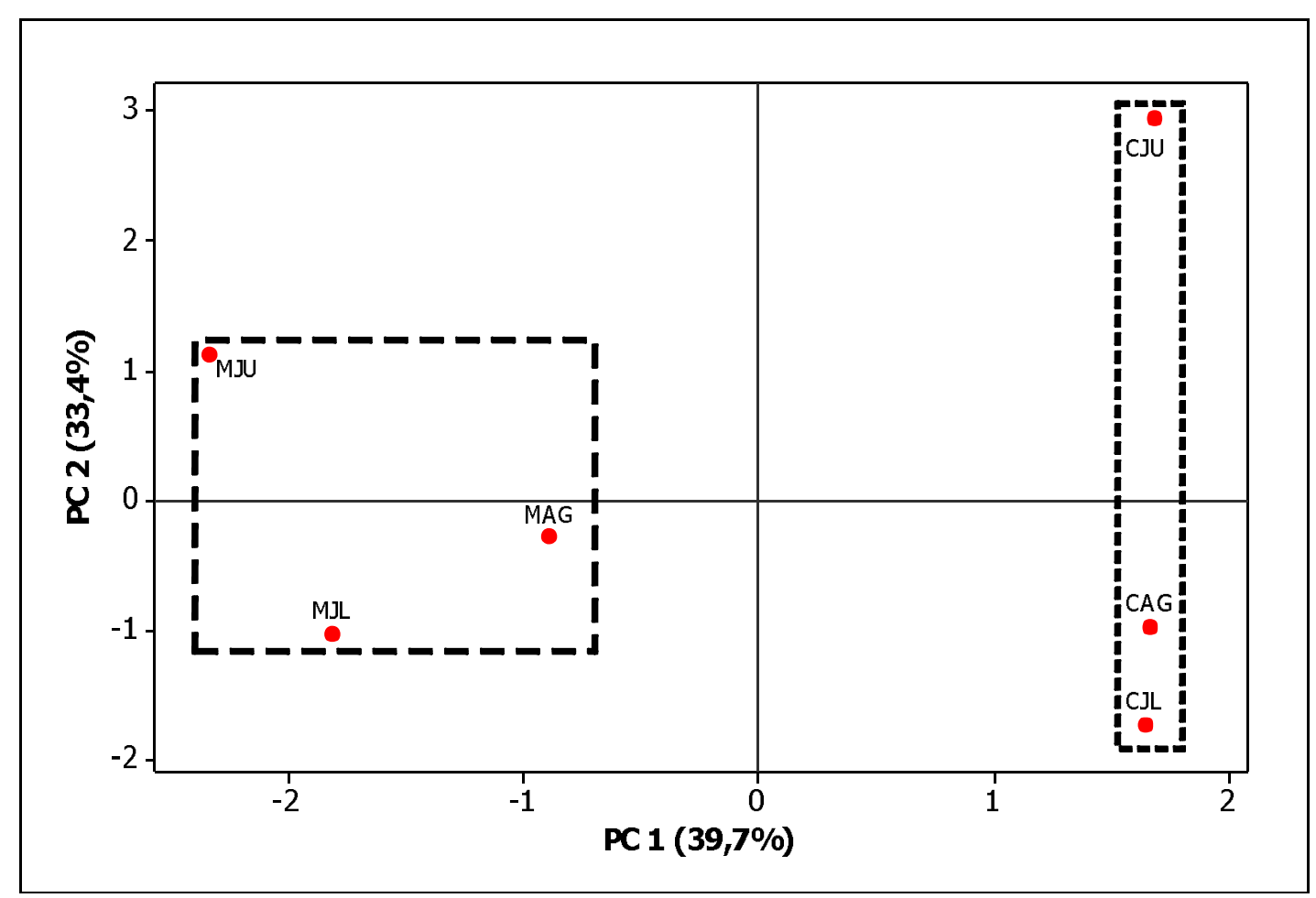

Figura 2: Gráfico dos scores PC1 x PC2 correspondente às amostras de águas subterrâneas estudadas das comunidades de Monsarás e Condeixa.

Analisando o gráfico de score observa-se a separação nítida de dois grupos, destacados pelas formas geométricas, denominados de grupos $\mathrm{M}$ e C. Os quais são formados pelos pontos referentes às comunidades de Monsarás (M) e Condeixa (C), apresentando uma diferença entre as amostras de águas subterrâneas analisadas, por meio das propriedades químicas distintas dos corpos hídricos de cada comunidade.

$\mathrm{O}$ gráfico de loading (Figura 3) contribui para uma interpretação e entendimento de quais variáveis analisadas mais contribuíram para a separação e formação desses grupos.

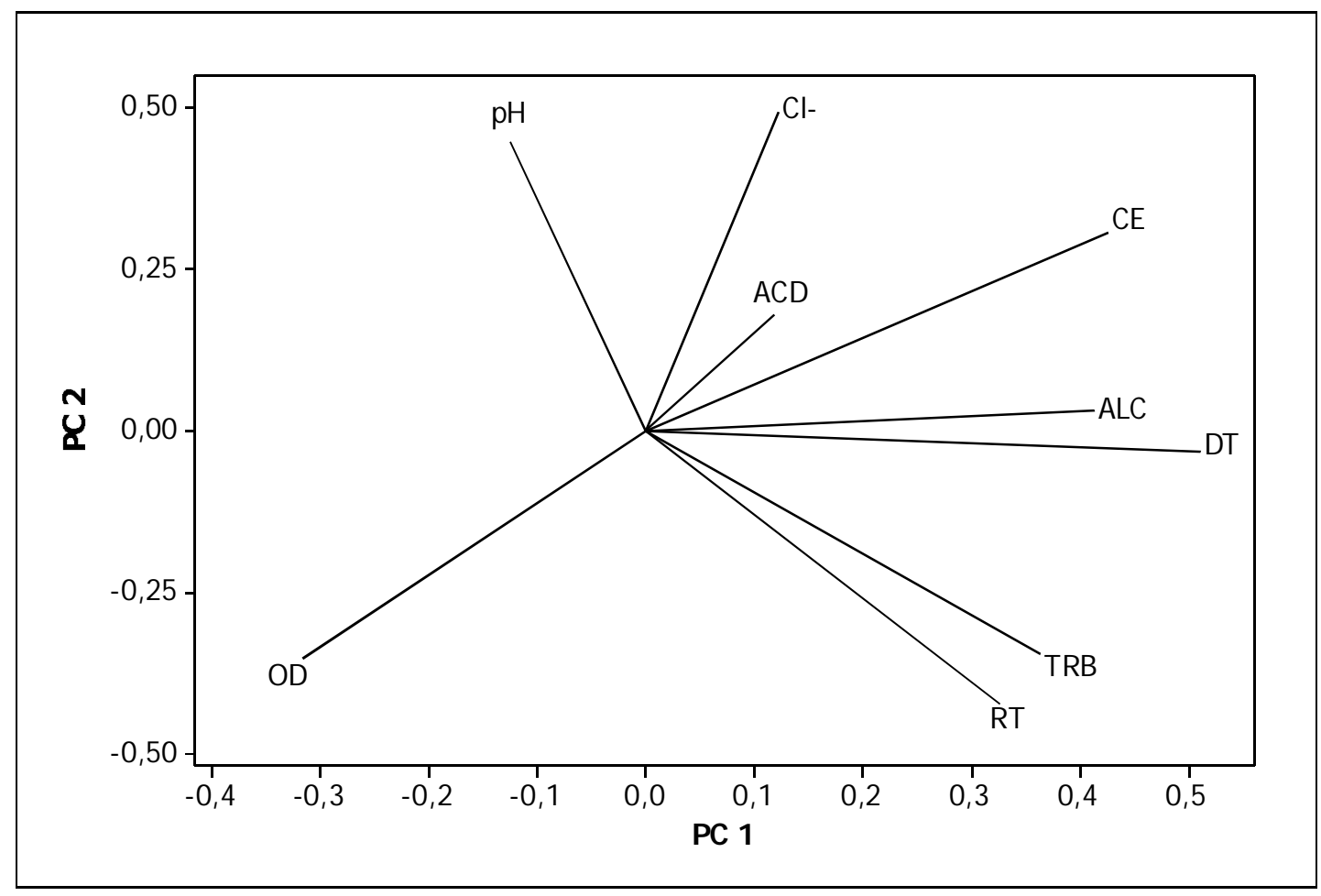

Figura 3: Gráfico dos loadings correspondentes aos parâmetros estudados nas amostras de águas subterrâneas de Monsarás e Condeixa: PC1 x PC2. 
No gráfico de loading observa-se que o potencial hidrogeniônico $(\mathrm{pH})$ e o oxigênio dissolvido (OD), foram os parâmetros que contribuíram de forma mais significativa para a formação do grupo referente à comunidade de Monsarás. Pelas análises físico-químicas observa-se que, para os meses analisados, o pH e o OD apresentaram os maiores valores. Assim, comprovando a separação em relação às amostras da comunidade de Condeixa, que apresentaram valores baixos em relação aos de Monsarás.

Por outro lado, as concentrações de $\mathrm{Cl}^{-}$, DT, ALC, ACD, TRB, CE e RT, foram os parâmetros que contribuíram de forma mais significativas para a formação e separação do grupo referente à comunidade de Condeixa. Situação caracterizada pelo fato desses parâmetros apresentarem maiores valores em relação aos resultados encontrados para Monsarás.

A Tabela 3 com os pesos dos coeficientes referentes aos parâmetros determinados permite analisar através de valores (pesos), quais desses parâmetros tiveram maior importância e se correlacionaram de forma positiva com as componentes principais mais significativas, ou seja, PC1, PC2 e PC3.

Tabela 3: Valores de loadings para as componentes principais, variância explicada e variância acumulada. Os valores mais significativos apresentam loading $>0,40$.

\begin{tabular}{c|c|c|c}
\hline Parâmetros & PC1 & PC2 & PC3 \\
\hline pH & $-0,126$ & $\mathbf{0 , 4 4 9}$ & 0,407 \\
\hline OD & $-0,318$ & $-0,352$ & 0,213 \\
\hline CE & $\mathbf{0 , 4 2 6}$ & 0,307 & 0,141 \\
\hline Acidez (ACD) & 0,118 & 0,181 & $\mathbf{0 , 6 5 1}$ \\
\hline Alcalinidade (ALC) & 0,414 & 0,031 & $-0,459$ \\
\hline Cloreto (Cl') & 0,122 & $\mathbf{0 , 4 9 6}$ & $-0,198$ \\
\hline Dureza Total (DT) & 0,512 & $-0,031$ & 0,110 \\
\hline Turbidez (TRB) & 0,364 & $-0,346$ & 0,236 \\
\hline Resíduos Totais (RT) & 0,327 & $-0,424$ & 0,167 \\
\hline Variância explicada (\%) & 39,7 & 33,4 & 19,8 \\
\hline Variância acumulada (\%) & 39,7 & 73,1 & 92,9 \\
\hline
\end{tabular}

$\mathrm{Na}$ PC1, que explicou aproximadamente $39,7 \%$ da variância dos dados, foi destacado em negrito o valor de significância da CE $(0,426)$ e na PC2, que explicou cerca de 33,4\% da variação dos dados, o valor de $\mathrm{Cl}^{-}(0,496)$. Esses parâmetros apresentaram no mês de junho valores muito elevados nas amostras de água da comunidade de Condeixa. Fato esse responsável pelo comportamento do ponto CJU em relação ao outros meses (CJL e CAG) observado no gráfico de scores (Figura 2).

$\mathrm{Na}$ PC2 também foi destacado o peso referente ao $\mathrm{pH}$ $(0,449)$ e na PC3, que explicou $19,8 \%$ da variação dos dados, foi destacado o peso da $\operatorname{ACD}(0,651)$. Esses parâmetros são responsáveis pelo comportamento do ponto MAG, que se distanciou em relação aos demais meses na comunidade de Monsarás (MJU e MJL), por apresentar os menores valores.

Os resultados observados na projeção das componentes principais $\mathrm{PC} 1$ x PC2, por meio da associação entre os parâmetros determinados, são confirmados na análise hierárquica de agrupamento (HCA).

Empregando-se a padronização dos dados, ligações completas e distancia euclidiana, obteve-se o dendograma dado na Figura 4.

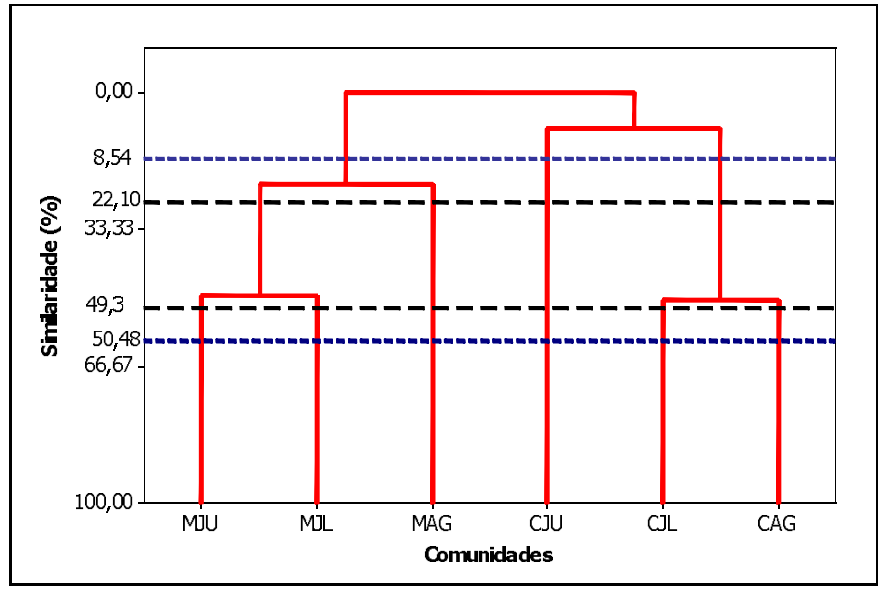

Figura 4: Dendograma para as análises físico-químicas e químicas das amostras de águas das comunidades de Monsarás e Condeixa. 
No dendograma observa-se o agrupamento de dois grandes grupos, comunidade de Monsarás (MJU, MJL e MAG) e Condeixa (CJU, CJL e CAG), os quais não apresentam similaridades. As linhas horizontais tracejadas correspondem às similaridades dos agrupamentos formados. As linhas horizontais tracejadas, na cor preta, são referentes ao grupo de Monsarás, para o qual foram destacadas duas similaridades, observa-se que dentro do grupo ocorreu uma separação entre o ponto MAG e os pontos MJU e MJL, explicada anteriormente pela contribuição dos parâmetros $\mathrm{pH}$ e ACD.

No entanto, esta separação não promoveu dissimilaridade do ponto MAG com os demais, apresentando uma similaridade de aproximadamente $22,10 \%$ com os pontos MJU e MJL. Os pontos MJU e MJL apresentaram uma similaridade de aproximadamente 49,36\%, por apresentarem nesses meses médias para os parâmetros determinados muito próximas. As similaridades apresentadas por este agrupamento é explicada pelo fato das amostras analisadas pertencerem ao corpo hídrico da mesma comunidade.

Para o agrupamento referente à comunidade de Condeixa, suas similaridades são representadas pelas linhas horizontais tracejadas na cor azul. Analisando da esquerda para direita é notória a separação do ponto CJU, fato esse explicado pela contribuição dos parâmetros $\mathrm{CE}$ e $\mathrm{Cl}^{-}$. O que proporciona uma baixa similaridade $(8,54 \%)$ com relação aos pontos CJL e CAG. Por outro lado, a similaridade entre os pontos CJL e CAG é de aproximadamente $50,48 \%$. Sendo suas similaridades explicadas por pertencerem ao mesmo corpo hídrico.

\section{CONCLUSÃO}

Os resultados obtidos para os parâmetros estabelecidos pela Portaria 2.914/2011 do Ministério da Saúde mostraram que existe restrição à potabilidade por conta das concentrações elevadas da turbidez na comunidade de Condeixa, devido à ausência de proteção dos poços contra a erosão e deposição de partículas em suspensão. No entanto os demais parâmetros encontram-se em conformidade com a portaria. Os parâmetros não preconizados pela legislação contribuíram de forma relevante para estabelecer a qualidade quanto à contaminação proveniente de ações antrópicas. A análise de componentes principais levou a separação de dois grupos de amostras ( $\mathrm{M}$ e $\mathrm{C})$. A combinação das PCs mais significativas explicou $92,8 \%$ das variações dos dados, sendo que a PC1 $(39,7 \%)$ e a PC2 $(33,4 \%)$ apresentaram maior percentual quanto a descriminação das amostras analisadas.

$\mathrm{Na} \mathrm{PC} 1$, o peso da variável condutividade elétrica juntamente com a concentração de íons de cloreto na PC2, por apresentarem as maiores concentrações registrados na comunidade de Condeixa, em especifico, no mês de junho, foram os parâmetros que contribuíram de forma mais significativa para a baixa similaridade com os demais meses $(8,54 \%)$, caracterizando uma alta quantidade de íons dissolvidos nas amostras analisadas no mês de junho. $\mathrm{Na} \mathrm{PC} 2$, o peso da variável $\mathrm{pH}$ em conjunto com as concentrações de acidez na PC3, por apresentarem os valores mais baixos na comunidade de Monsarás, em especifico, no mês de agosto, favoreceram a baixa similaridade $(22,10 \%)$ com os outros meses que apresentaram uma similaridade de 49,36\%.

Assim, a utilização da análise multivariada contribuiu na interpretação dos dados e na obtenção de informações importantes para identificação de semelhanças e diferenças presentes nas amostras de águas subterrâneas relacionadas aos parâmetros estudados.

\section{REFERENCIAS}

[1] CHAPLIN, M. F. Water: its important to life. Biochemistry and Molecular Biology Education. p. 54-59. 2001. Acesso em: 16 de novembro de 2016. Disponível em:

http://w3.ualg.pt/ pmartel/cadeiras/be/papers/4.pdf.

[2] MESQUITA, F. R., et al. Análise Físico-Química e Microbiológica da Água: Estudo de Caso no Balneário Igarapé Preto, Cruzeiro do Sul, Acre, Brasil. Enciclopédia Biosfera, Centro Cientifico Conhecer, Goiânia, v.10, n.19, p. 2676, out/dez. 2014.

[3] PATOLE, M. Brazilian Groundwater Law - Abstraction and Pollution Controls, 2015. Acesso em: 15 de novembro de 2016.

Disponível

em:https://www.researchgate.net/publication/288494232_Brazilia n_Groundwater_Law_-_Abstraction_and_Pollution_Controls. DOI: 10.13140.

[4] United Nations Environment Programme - UNEP. Status Report on The Application of Integrated Approaches to Water Resources Management. 2012. ISBN: 978-92-807-3264-1. Acesso em: 15 de novembro de 2016. Disponível em: http://www.unwater.org/downloads/UNW_status_report_Rio2012 .pdf.

[5] ZAN, R. A., et al. Análise Microbiológica de Amostras de Água de Poços Rasos Localizados no Município de Buritis, Região do Vale a Jamari, Rondônia, Amazônia Ocidental. 2012, v.8, n. 8, p. 1867-1875. Acesso em: 15 de novembro de 2016. Disponível

em: https://periodicos.ufsm.br/reget/article/view/7293/pdf. ISSN: 2236-1170. DOI: 10.5902/223611707293.

[6] The Food and Agriculture Organization of the United Nations and Earthscan (FAO). The state of the world's land and water resources for food and agriculture (SOLAW) - Managing systems at risk. Food and Agriculture Organization of the United Nations, Rome and Earthscan, London. 2011. Acesso em: 15 de novembro de 2016.2 Disponível em: http://www.fao.org/docrep/017/i1688e/i1688e.pdf. ISBN: 978-925-106614-0(pbk).

[7] ZEKTSER, I. S.; EVERETT, L. G. Groundwater Resources of The World and Their Use. Series on groundwater. 2004, n.06. Acesso em: 16 de novembro de 2016. Disponível em: http://unesdoc.unesco.org/images/0013/001344/134433e.pdf. ISBN 92-9220-007-0.

[8] UNESCO. Internationally Shared (Transboundary) Aquifer Resources Management Their significance and sustainable management. 2001. Acesso em: 15 de novembro de $2016 . \quad$ Disponível http://unesdoc.unesco.org/images/0012/001243/124386e.pdf.

[9] ANWAR, K. M.; VANITA, A. Analysis Of Groundwater Quality Using Statistical Techniques: A Case Study Of Aligarh City (India). International Journal of Technical Research and Applications v. 2, n. 5, p. 100-106. 2014. Acesso em: 15 de novembro de 2016. Disponível em: http://www.ijtra.com/view/analysis-ofgroundwater-quality-using-statistical-techniques-a-case-study-ofaligarh-city-india.pdf. ISSN: 2320-8163. 
[10] DOHARE, D.; DESHPANDE, S.; KOTIYA, A. Analysis of Ground Water Quality Parameters: A Review. Research Journal of Engineering Sciences. v. 3, n. 5, p. 26-31. 2014. Acesso em: 13 de novembro de 2016. Disponível em: http://www.isca.in/IJES/Archive/v3/i5/3.ISCA-RJEngS-2014-

24.pdf. ISSN $2278-9472$.

[11] BRASIL. Ministério da Saúde. Portaria $\mathrm{n}^{\circ}$ 2914, de 12 de dezembro de 2011. Acesso em: 5 de novembro de 2016. Disponível em:

http://site.sabesp.com.br/uploads/file/asabesp_doctos/kit_arsesp_p ortaria2914.pdf.

[12] IBGE. Instituto Brasileiro de Geografia e Estatística, 2010. Acesso em: 16 de outubro de 2016. Disponível em: http://www.ibge.gov.br/estadosat/perfil.php?sigla=pa.

[13] APHA (American Public Health Association). Standard Methods for the Examination of Water and Wastewater. American Water Works Association (AWWA) and Water Environment Federation (WEF), 21a. ed., Washington, DC: USA, 2005.

[14] BURGIN, A.; GROFFMAN, P.M. Soil O2 controls denitrification rate and $\mathrm{N}_{2} \mathrm{O}$ yield in a riparian wetland. Journal of Geophysical Research 117: G01010. 2012. Acesso em: 17 de novembro de 2016. Disponível em: http://onlinelibrary.wiley.com/doi/10.1029/2011JG001799/full. DOI: $10.1028 / 2011 J G 001799$.

[15] VIANA, M. S., et al. Qualidade Físico-Química das Águas para Abastecimento Humano no Município de Manhumirim (MG). Revista Científica da Faminas. Minas Gerais, v. 6, n. 3, set/dez. 2010.

[16] UMAR, M., et al. The Impact of Geology of Recharge Areas on Groundwater Quality: A Case Study of Zhob River Basin, Pakistan. 2012, 41 (2), 119-127. Acesso em: 18 de novembro de $2016 . \quad$ Disponível em: http://onlinelibrary.wiley.com/doi/10.1002/clen.201100581/full. DOI: $10.1002 /$ clen.201100581. 